\title{
Computational Investigation of Flow Separation over Naca 23024 Airfoil at 6 Million Free Stream Reynolds Number
}

\author{
B. S. Anil Kumar ${ }^{1}$, Ramalingaiah ${ }^{2}$, S. Manjunath ${ }^{3}$, Rudresh Ganganna ${ }^{4}$ \\ ${ }^{1}$ Department of Mechanical Engineering, BNMIT, Visveswaraya Technological University, Bangalore, India \\ ${ }^{2}$ Department of Mechanical Engineering, PESCE, Visveswaraya Technological University, Mandya, India \\ ${ }^{3}$ Department of Mathematics, BNMIT, Visveswaraya Technological University, Bangalore, India \\ ${ }^{4}$ Niharika Institute of Computational Engineering (NICECFD), Bangalore, India
}

\section{Email address:}

anilbs_phd@yahoo.com (B. S. A. Kumar), aniram161957@gmail.com (Ramalingaiah), drmanjus@gmail.com (S. Manjunath), nicecfd@rediffmail.com (R. Ganganna)

\section{To cite this article:}

B. S. Anil Kumar, Ramalingaiah, S. Manjunath, Rudresh Ganganna. Computational Investigation of Flow Separation over Naca 23024 Airfoil at 6 Million Free Stream Reynolds Number. International Journal of Science, Technology and Society. Vol. 3, No. 6, 2015 , pp. $315-321$. doi: $10.11648 /$ j.ijsts.20150306.17

\begin{abstract}
The following work is the CFD analysis of NACA 23024 airfoil. The analysis is carried out for a free stream Reynolds number of 6 million for which the wind tunnel results are available. The CFD analysis is carried out using Ansys Fluent Solver. The analysis is carried out using Spalart Allmaras turbulence model, K-omega SST turbulence model with flow transition capabilities, Standard K-Epsilon Turbulence model and K-omega SST turbulence model.It is to be noted that each turbulence model employs different mathematical approach to model boundary layer. The analysis results are then compared with the wind tunnel results and the performance of the turbulence models are discussed. This study recommends an accurate methodology to conduct CFD analysis for external aerodynamic flows.
\end{abstract}

Keywords: NACA 23024, Turbulence Models, Lift Curve, Drag Curve, Stalling

\section{Introduction}

The CFD analysis on the chosen airfoil NACA 23024, where first digit when multiplied by $3 / 2$ yields the design lift coefficient in tenths of chord, the next two digits when divided by 2 gives the position of the camber in tenths of chord and the final two digits indicate the maximum thickness in percentage of chord that is NACA 23024 airfoil has maximum thickness of $24 \%$, a design lift coefficient of $(2 \times 3 / 2) 3$ in tenths and maximum camber located (30/2) 15\% back from the leading edge is carried out using the ANSYS package. ICEM CFD is used as a meshing tool where a mesh required for the analysis of the airfoils are developed. The mesh s adjusted to obtain a $y$-plus close to 30 so that the best approximation is achieved. In operating conditions, the wing section that is the airfoil of the aeroplane moves through the wind. However, for simulation and experimental purposes the airfoil is kept stationary and the working fluid is assumed to have motion over the airfoil. Hence, a circular far field is created to obtain the best approximation.

The actual solving of the problem is done using the Fluent package which is a part of the ANSYS package. The case is analysed as an incompressible low regime problem. In the physics setup, the atmospheric pressure is input as absolute pressure. There are no temperature inputs as it is an incompressible flow. The input boundary condition is the velocity inlet boundary condition and the output boundary condition is the outflow type boundary condition. A good convergence criteria is set to make the results more accurate.

\section{Experimental Methods v/s CFD}

The traditional approach of product design involves the fabrication of physical prototype, conducting experimental test on the prototype and modifying the design till it meets the requirements. Unfortunately, the traditional approach is time consuming and it is difficult to build physical Prototypes during the early stages of the design. The construction and testing of many prototypes is often needed to meet a stringent design requirement. This can turn into an expensive process with the potential to delay the entire development cycle. Another problem is that although 
building and testing of prototypes can yield accurate performance measurements, it sheds little light on the internal flow conditions that determine why the design does or does not work. As a result, engineers obtain very little information from each test and have to proceed mainly on instinct. In order to address these issues, engineers have been using computer simulations for a number of years to create virtual prototypes of their concept designs and evaluate their design without the time and expense required for physical prototyping. Computational fluid dynamics (CFD) software allows users to build models that simulate the flow conditions, making it possible to evaluate virtual prototypes on a computer. Virtual prototyping can be performed at a much lower cost and in much less time than physical prototyping, and has the additional advantage that engineers can determine important flow variables, such as velocity, pressure, and temperature at any point in the design, making it easier to optimize the design.

Experimental techniques provide the most reliable information regarding flow characteristics and performance of any system. It is quite valuable in validating mathematical solutions of the governing equations of flow. Accurate data can be obtained by conducting full scale testing at actual operating conditions provided the measuring equipment has good precision and accuracy. But in large complex systems like combustor and turbo machines of aero-engines, tunnel size and the difficulty in adequately simulating the prototype flow field, makes experimental fluid mechanics an impracticable means of obtaining flow fields for many problems. On the other hand the total expense associated with CFD capability is considerably lower than that of a high quality experimental facility.

\section{Geometry Creation and Mesh Generation}

1. The coordinates of the airfoil is obtained and is imported into ANSYS ICEM-CFD software, which is essentially a meshing tool. The curves option is checked while importing the points into CFD which draws splines between the points for the upper and lower surface.
2. The coordinates obtained are for an open trailing edge type airfoil and therefore, the two end points have to be joined using the curve option.

3. An exact circular far field is created around the airfoil equidistant from its chord in all the four directions.

4. After the far field has been generated, blocking is done. Blocking is essentially done to capture the shape of the far field of the airfoil and further splitting of the block is done to achieve the capturing of the airfoil

5. Once the splitting of the block is complete, point to point association of the main block is done along with the edge to curve association.

6. An O-grid is a tool which is available in the blocking option itself. This is created around the airfoil in order to obtain the mesh exactly in the shape of the airfoil surface around it.

7. An O-grid creates a localized block in an $\mathrm{O}$ shape around the airfoil. Edge to curve association is carried out in order to associate the O-grid with the airfoil. The link edge command is made use of to make the lines of the 0 -grid take the shape of the airfoil surface.

8. After all the associations are done, the pre-mesh parameters are applied to the edges. The selection of the meshing law and the number of nodes along with the ratios and spacing options play the most important part in the meshing process as they decide the quality of the mesh around the airfoil.

9. It is desirable to have more grid elements near the airfoil surface to increase computational accuracy and to have comparatively lesser number of grid elements away from the airfoil in the far field in order to reduce computational time and reduce the size of the output file. To achieve this requirement, the exponential 2 mesh law is used.

10. The y-plus is checked for after the meshing is complete and modified if necessary through the spacing option in the pre-mesh parameters.

11. After the entire mesh generation and obtaining a quality mesh, it is converted to unstructured mesh to make it possible to be read in Fluent. The file is saved in the output option to obtain a file which is compatible with Fluent. It is saved with the extension. msh.

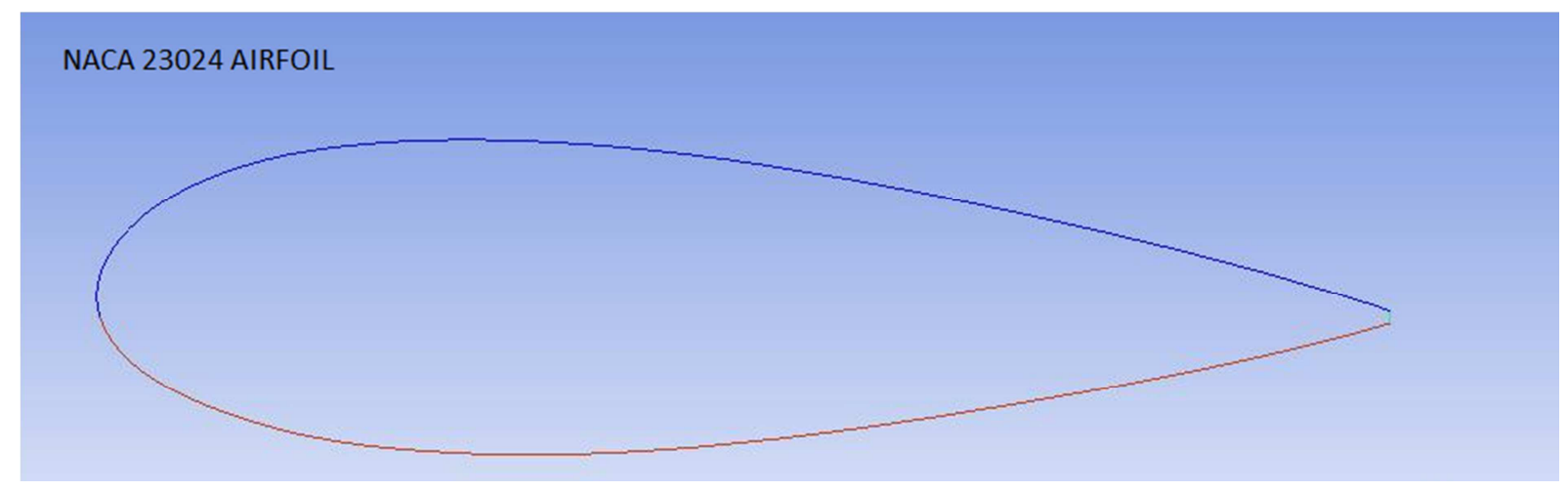

Figure 1. NACA 23024 airfoil. 


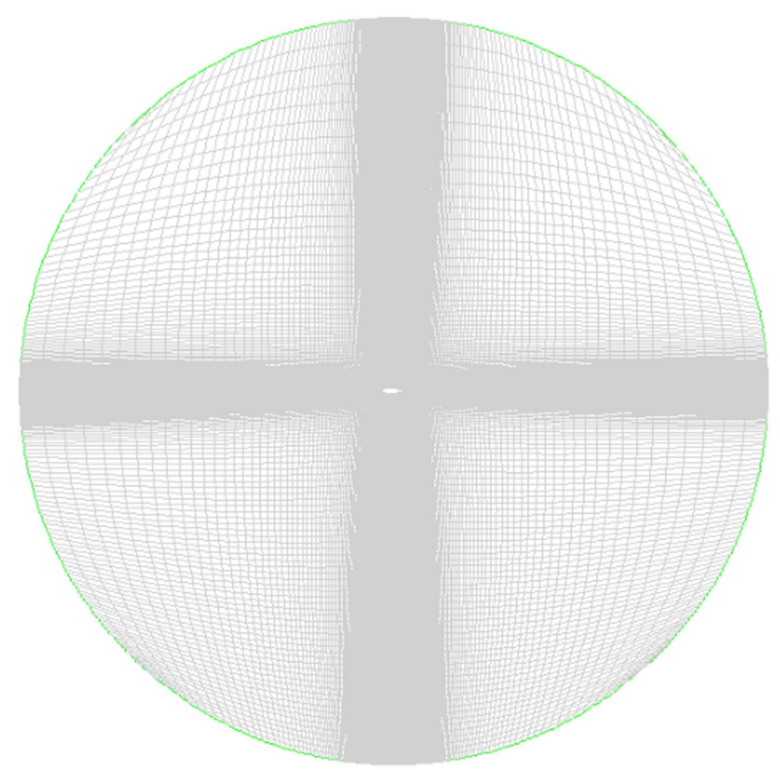

Figure 2. Mesh generation of NACA 23024 airfoil.

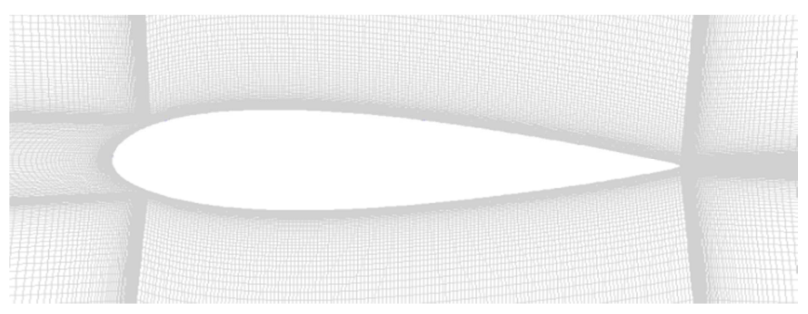

Figure 3. Mesh resolution in the boundary layer region.

Table 1. Comparison between CFD results vs. Wind tunnel test results.

\begin{tabular}{llllll}
\hline \multicolumn{2}{l}{ CFD analysis results } & \multicolumn{4}{c}{ Wind tunnel test results } \\
\hline $\mathbf{A O A}$ & $\mathbf{C}_{\mathbf{L}}$ & $\mathbf{C}_{\mathbf{D}}$ & $\mathbf{A O A}$ & $\mathbf{C}_{\mathbf{L}}$ & $\mathbf{C}_{\mathbf{D}}$ \\
\hline 0 & 0.11368 & 0.0096 & 0 & 0.1 & 0.0085 \\
2 & 0.30205 & 0.0101 & 2 & 0.3 & 0.009 \\
4 & 0.48780 & 0.0103 & 4 & 0.5 & 0.0095 \\
6 & 0.67509 & 0.0126 & 6 & 0.7 & 0.011 \\
8 & 0.87800 & 0.0139 & 8 & 0.9 & 0.012 \\
10 & 1.02000 & 0.0148 & 10 & 1 & 0.013 \\
12 & 1.16000 & 0.0169 & 12 & 1.18 & 0.015 \\
13 & 1.16000 & 0.0186 & 14 & 1.3 & 0.016 \\
14 & 1.12000 & 0.07169 & 16 & 1.15 & \\
15 & 1.10000 & & 18 & 1.11 & \\
16 & 1.00000 & & 20 & 1.05 & \\
17 & 0.96000 & & 22 & 1 & \\
18 & 0.89000 & & 24 & 0.98 & \\
19 & 0.84000 & & & & \\
20 & 0.76000 & & & & \\
\hline
\end{tabular}

The above table indicate the analysis carried out on NACA 23024 airfoil for a Reynolds number of 6 million using wind tunnel test results and CFD. The wind tunnel results are obtained from verified and published data. ( Theory of wing section by Abott)

CFD analysis is carried out using Ansys package and $\mathrm{C}_{\mathrm{L}}$ and $C_{D}$ values are directly obtained by the post processor. The airfoil is tested at $0^{0}$ to $20^{\circ}$ angle of attack (AOA) and the plot of $\mathrm{C}_{\mathrm{L}} \mathrm{V} / \mathrm{S}$ AOA and $\mathrm{C}_{\mathrm{D}} \mathrm{V} / \mathrm{S}$ AOA are obtained

It is clear from above two tables that the CFD results are very good agreement with the wind tunnel test data as a result of superior mesh quality.

\section{CFD Analysis in FLUENT Software}

1. The ANSYS fluent is opened and the option is set to 2D and double precision.

2. Import the mesh by selecting read mesh option. Scale the mesh to the suitable unit. The check command may be used to check for the quality of the mesh.

3. Fluent software used not only for aerodynamic studies but also used in heat transfer problems, acoustic problems etc. therefore, it is important to select the correct set of equation for the required analysis.

4. After the turbulence model is selected for the analysis, air is selected as the material and the corresponding material properties are input and saved.

5. For the cell zone boundary condition, the far field is selected to be a fluid and the material properties which were previously input are associated with the far field cell zones.

6. Input of the boundary condition is the most important part in the physics setup. Since it is an incompressible flow, the input boundary condition is the velocity type boundary condition where the magnitude of the free stream velocity is directly input. The outlet boundary condition is the outflow type boundary condition as the airfoil functions in atmosphere and there is no external pressurization.

7. The far field is set to the pressure far field boundary condition where the pressure is input as the atmospheric pressure at the location.

8. The solution method is set to simple. The monitors are activated by selecting the coefficient of lift, coefficient of drag.

9. The solution initialization is done by setting it to hybrid initialization and the residual monitors are edited to have convergence criteria to obtain more accurate results.

10. The run calculation command is selected and 2000 iterations are allowed. The calculation is run and it terminates automatically depending on the input convergence criteria or it may be manually interrupted in case the coefficient of lift and coefficient of drag become constant all the remaining iterations.

11. The required plots such as the $\mathrm{Cp}$ plot and required contours such as pressure and velocity vectors are obtained as the post processor results.

\section{Results and Discussions}

Figure 4 and 5 shows the lift and drag coefficients respectively for 6 million Reynolds number, plotted along 
with wind tunnel results.

Figure 9 shows the pressure coefficient plot for angle of attack 15 degree with Spalart allmaras turbulence model. Stalling is clearly seen.

Spalart allmaras turbulence model does not employ any approximations in the boundary layer region. The boundary layer region need to be resolved with very fine layer of mesh elements (10 to 15 layers). All the three regions of the turbulent boundary layer will be actually captured on the fine mesh near the wall, which is more realistic.

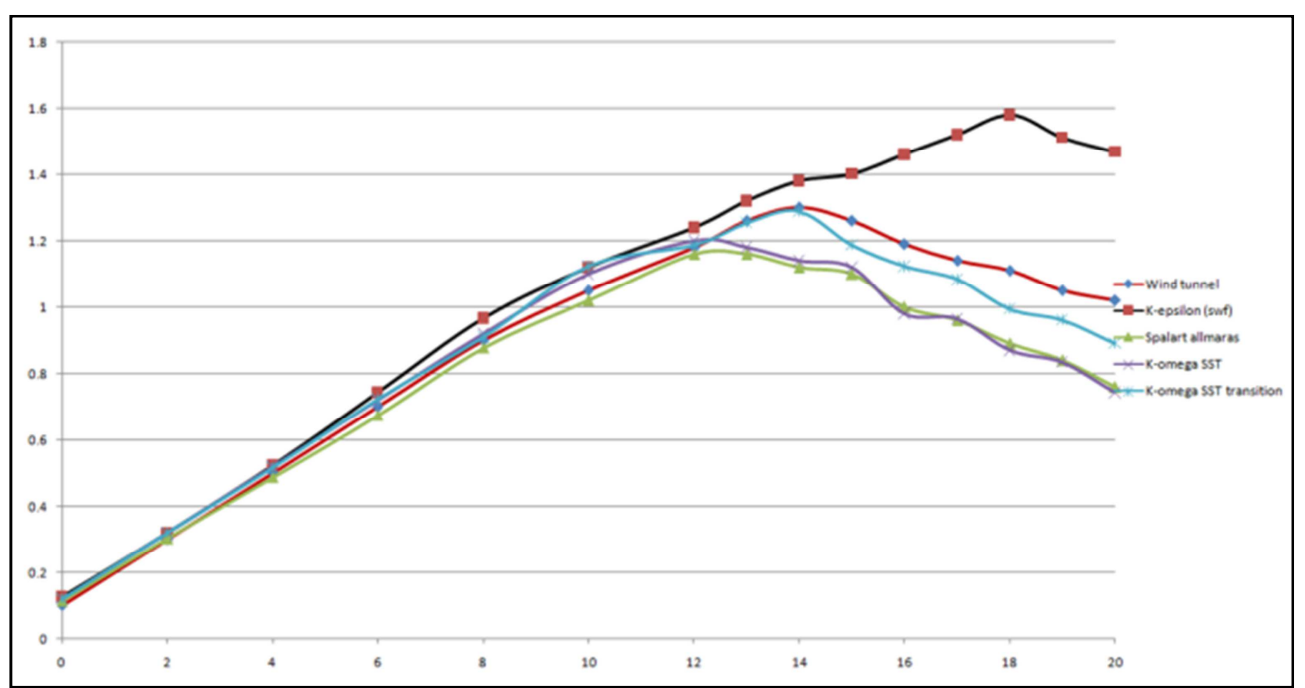

Figure 4. Lift curve.

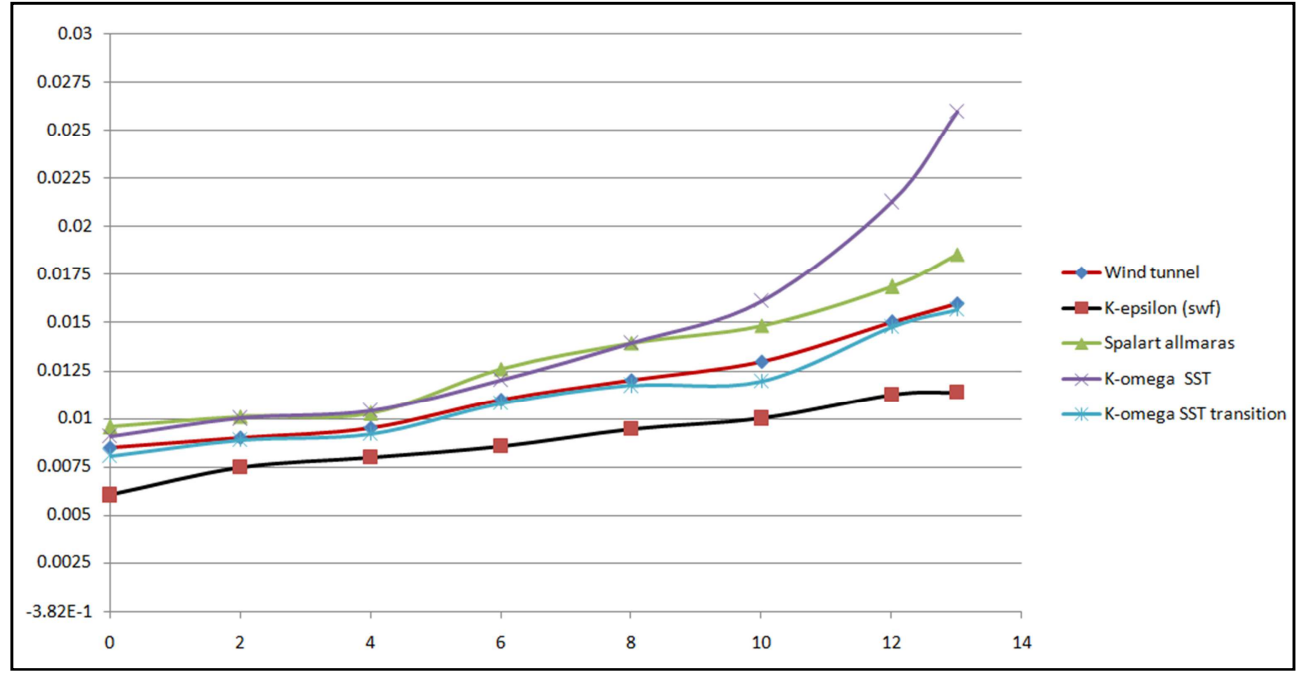

Figure 5. Drag curve.
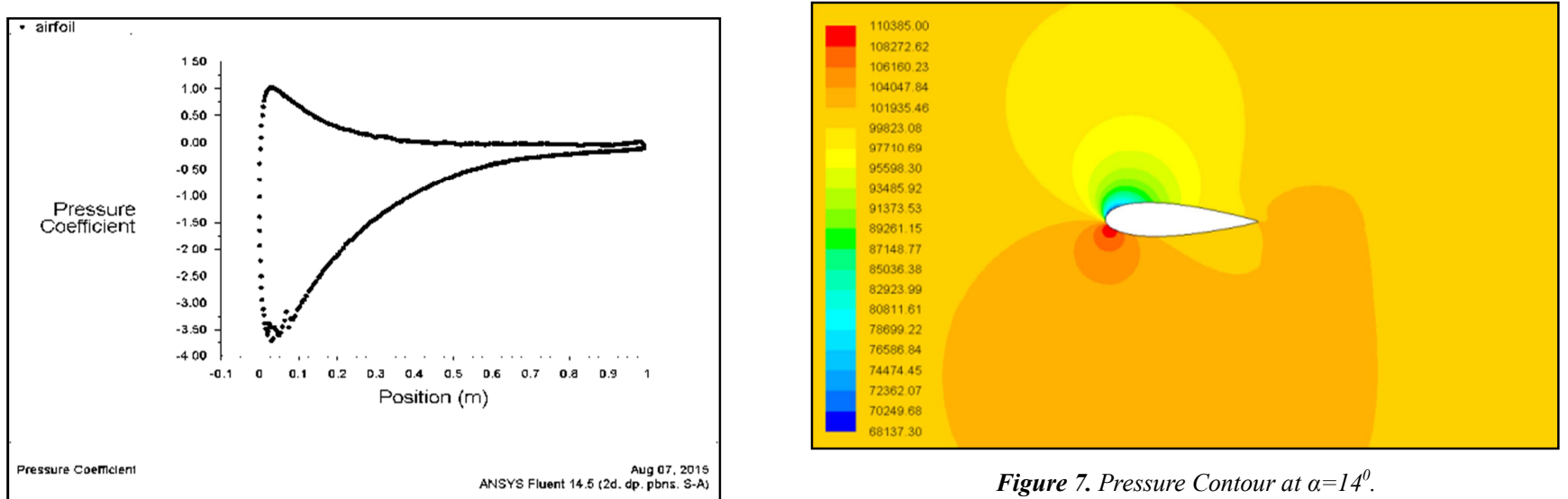

Figure 7. Pressure Contour at $\alpha=14^{0}$.

Figure 6. Pressure coefficient at $\alpha=14^{0}$. 


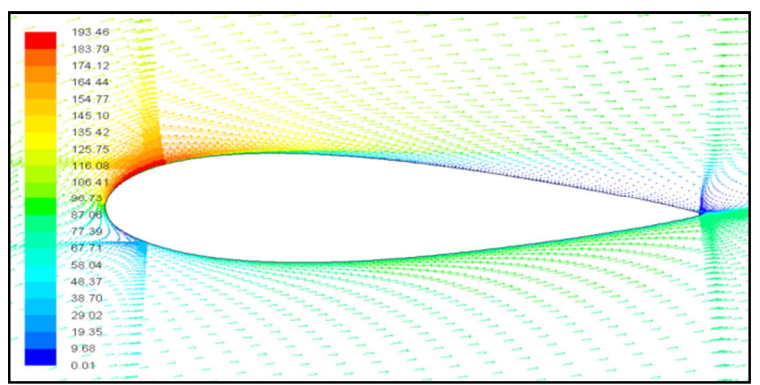

Figure 8. Velocity Vector at $\alpha=14^{0}$

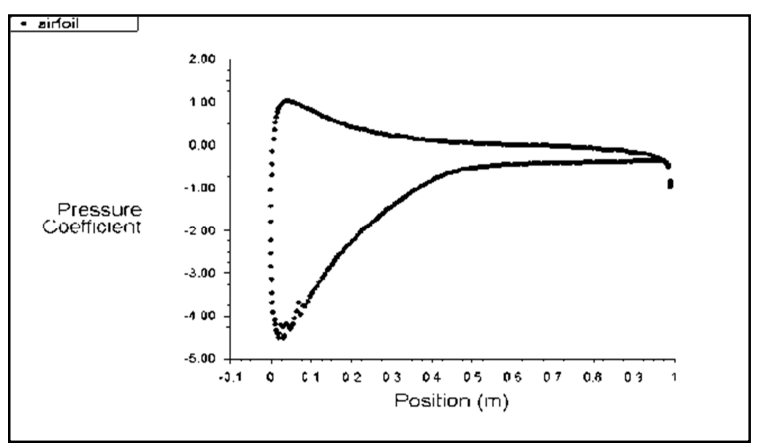

Figure 9. Pressure coefficient at $\alpha=15^{\circ}$.

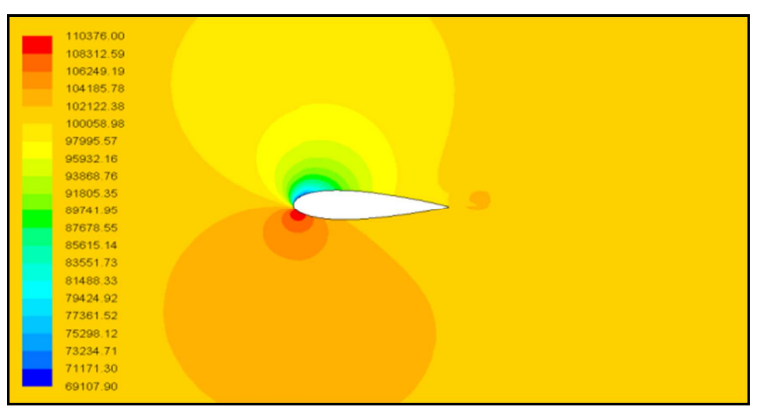

Figure 10. Pressure Contour at $\alpha=15^{\circ}$

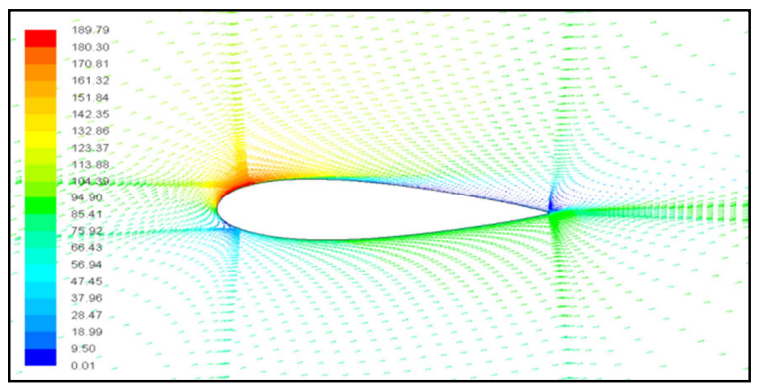

Figure 11. Velocity Vector at $\alpha=15^{\circ}$.

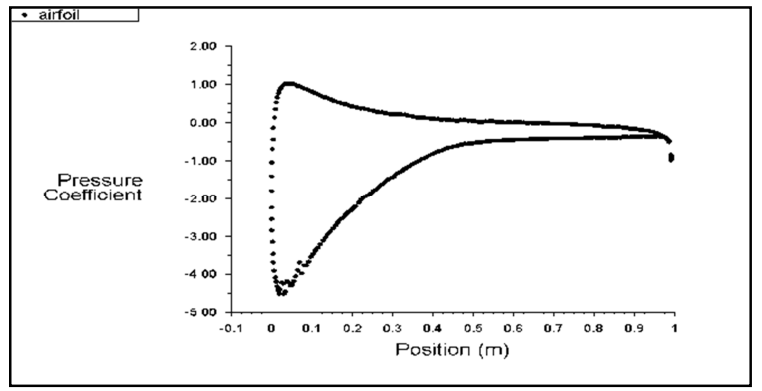

Figure 12. Pressure coefficient at $\alpha=16^{0}$.

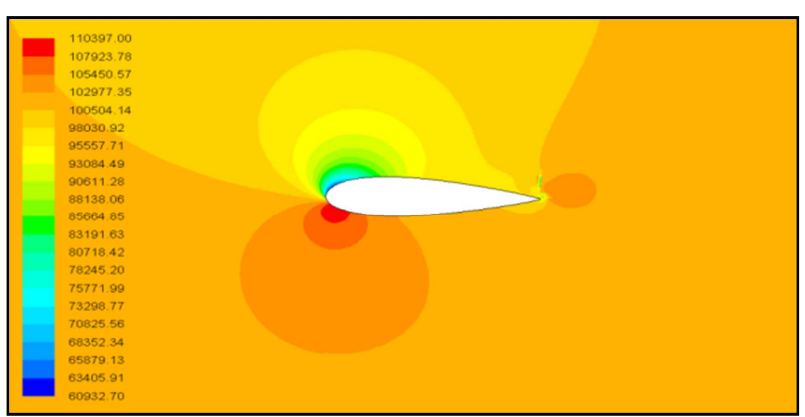

Figure 13. Pressure Contour at $\alpha=16^{\circ}$.

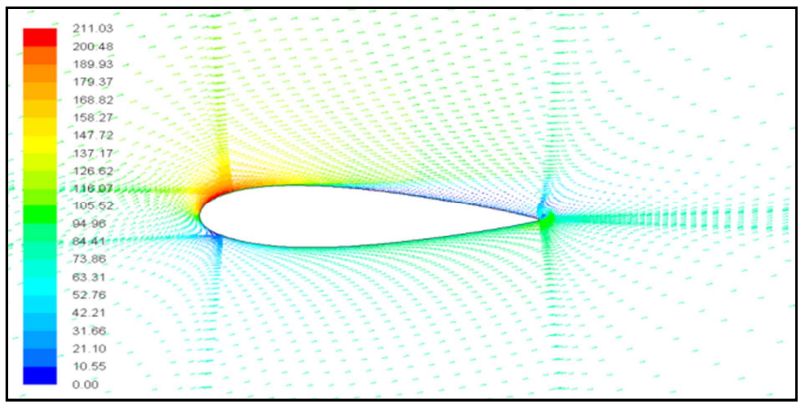

Figure 14. Velocity Vector at $\alpha=16^{\circ}$.

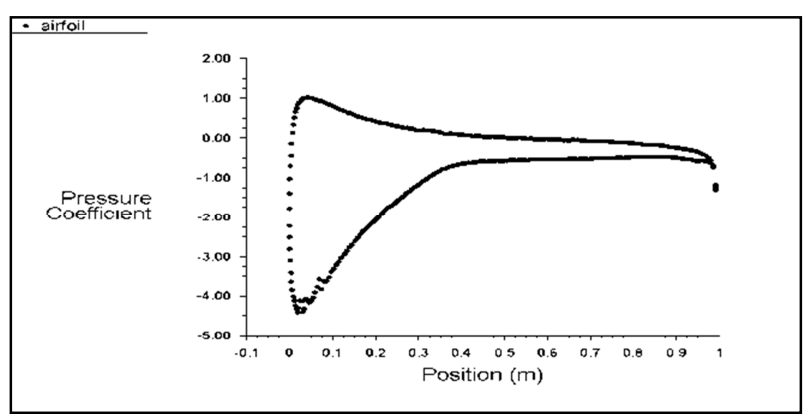

Figure 15. Pressure coefficient at $\alpha=17^{0}$.

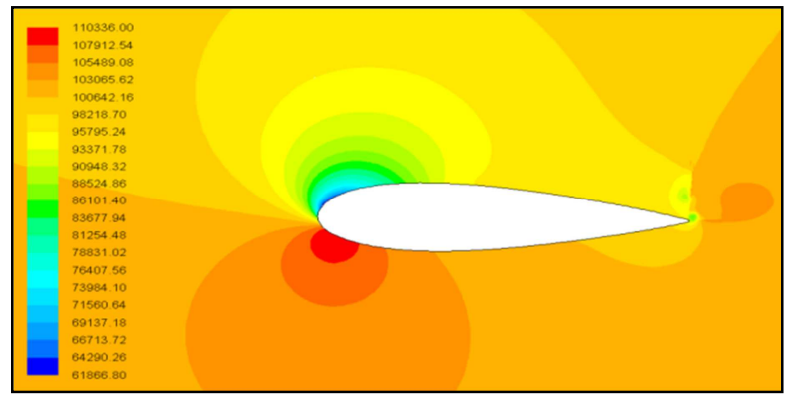

Figure 16. Pressure Contour at $\alpha=17^{\circ}$.

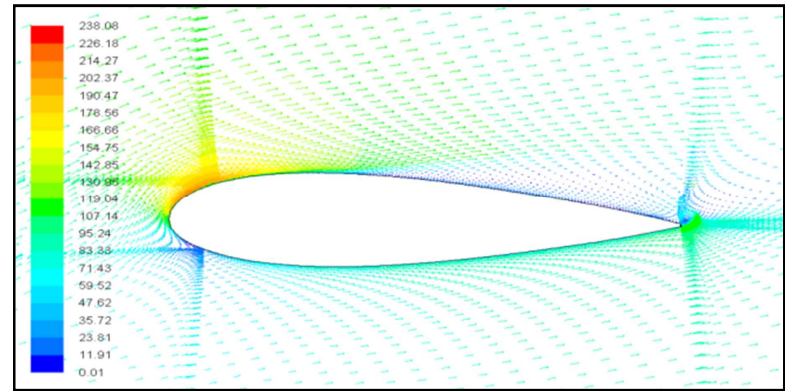

Figure 17. Velocity Vector at $\alpha=17^{\circ}$. 


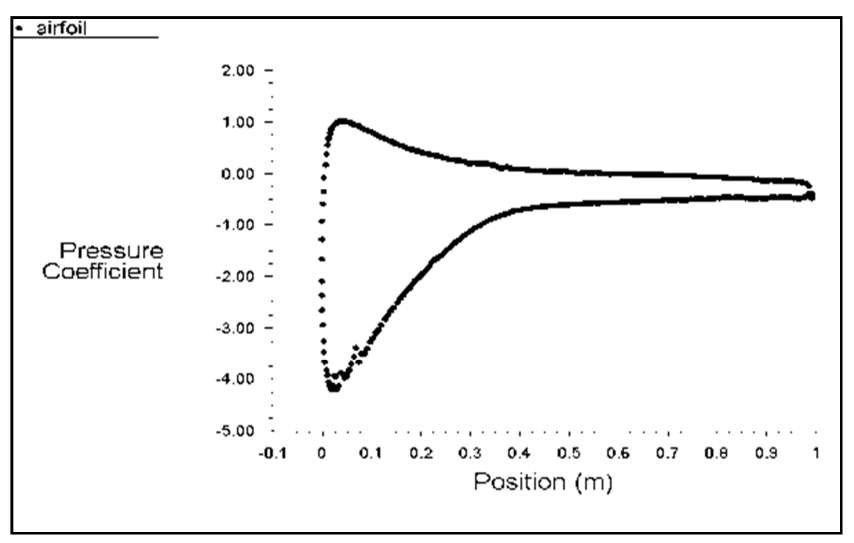

Figure 18. Pressure coefficient at $\alpha=18^{\circ}$.

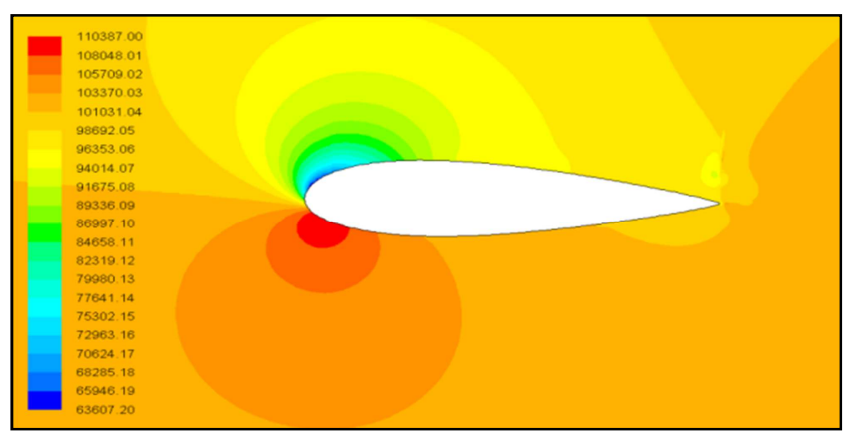

Figure 19. Pressure Contour at $\alpha=18^{\circ}$.

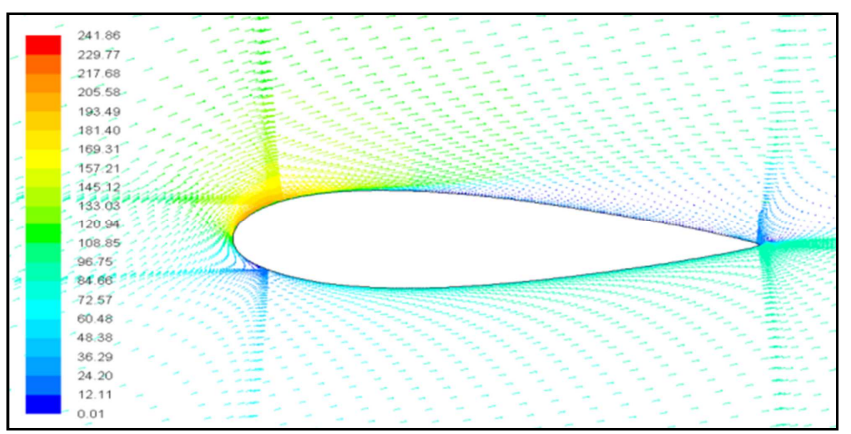

Figure 20. Velocity Vector at $\alpha=18^{0}$.

\section{Conclusions}

- All turbulence models yield similar results in the pre stall region and the CFD analysis results are providing a good match with wind tunnel test results.

- In the post stall region approximation of boundary layer regions with wall functions as in the case of K-epsilon turbulence model with standard wall functions, leads to delayed prediction of stalling and hence lift forces are over approximated.

- Capturing of all the three regions of the turbulent boundary layer with a very fine mesh layers will provide accurate CFD analysis results and trend same as that of wind tunnel test results.

- Prediction of both laminar and turbulent boundary layer regions with transition turbulence model provides most accurate CFD analysis results, and these results are very close to wind tunnel measurements.

\section{Acknowledgements}

Authors would like to express sincere gratitude to "Niharika Institute of Computational Engineering (NICECFD), Bangalore. (www.nicecfd.com) for all the technical guidance and licensed version of ANSYS ICEMCFD meshing software and ANSYS FLUENT CFD analysis software

\section{References}

[1] David Hartwanger et.al "3 D modeling of a Wind Turbine using CFD” NAFEMS Conference, United Kingdom, 2008.

[2] Frank Bertagnolio et.al "Wind Turbine airfoil catalogue" RISOE National Laboratories, Denmark, 2001.

[3] H. Gao et.al "Computational study of unsteady flows around dragonfly and smooth airfoils at low Reynolds number" $46^{\text {th }}$ AIAA Aerospace sciences meeting and exhibit, Reno, Navada, 2008.

[4] Vance Dippold, III, "Investigation of Wall Function and Turbulence Model Performance within the Wind Code", 43rd AIAA Aerospace Sciences Meeting and Exhibit, 10 - 13 January 2005, Reno, Nevada.

[5] S. Sarada, M. Shiva Shankar and Rudresh Ganganna "Numerical simulation of Viscous, incompressible flow around NACA 64618 subsonic airfoil using Computational Fluid Dynamics", Proceedings of National conference on advances in Mechanical Engineering, Allied Publishers Pvt Ltd, 2012.

[6] Menter, F. R., "Two-Equation Eddy-Viscosity Turbulence Models for Engineering Applications" AIAA Journal, Vol. 32, No. 8, August 1994, pp. 1598-1605 (http://turbmodels.larc.nasa.gov/sst.html).

[7] Abbott. I.H, "Theory of wing section, including a summary of airfoil data", Dover book on Physics, 1995.

[8] Menter FR, 1994, Two Equation Eddy-viscosity turbulence model for Engineering Applications, AIAA journal, USA, Volume 32, No.8, pp. 1598-1605.

[9] Drishtysingh Ramdenee, H. Ibrahim, N. Barka, A. Ilinca, 2013, Modeling of aerodynamic flutter on a naca 4412 airfoil wind blade, International Journal of Simulation and Process Modelling, Inderscience Publishers, Canada, Volume 8, No. 1 , pp. 79-87.

[10] Douvi C. Eleni, Tsavalos I. Athanasios and Margaris P. Dionissios, Evaluation of the turbulence models for the simulation of the flow over a National Advisory Committee for Aeronautics (NACA) 0012 airfoil, Department of Mechanical Laboratory, University of Patras, 26500 Patras, Greece, Journal of Mechanical Engineering Research Vol 4(3), pp. 100-111, March 2012 ISSN 2141-2383.

[11] Bacha WA, Ghaly WS (2006). Drag Prediction in Transitional Flow over Two-Dimensional Airfoils, Proceedings of the 44th AIAA Aerospace Sciences Meeting and Exhibit, Reno, NV.

[12] Menter FR (1994). Two-Equation Eddy-Viscosity Turbulence Models for Engineering Applications. AIAA J., 32: 1598-1605.

[13] Spalart, PR, Allmaras SR (1992). A One-Equation Turbulence Model for Aerodynamic Flows. AIAA Paper, pp. 92-439. 
[14] Michealamitay, Douglas R Smith, Valdis Kibens, David E. Parekh and Ali Glezer. "Aerodynamic flow control over unconventional airfoil using synthetic jet actuators". AIAA journal. V39, no1, march 2001.
[15] Gaurav Saxena, Mahendra Agarwal. "Aerodynamic analysis of NACA 4412 airfoil using CFD". International journal of emerging trends in engineering and development. Issue 3, vol 4, july 2013 . 